\title{
A RIGID BOREL SPACE
}

\author{
BOHDAN ANISZCZYK
}

(Communicated by Andrew M. Bruckner)

ABSTRACT. We construct a rigid $\sigma$-algebra on a set of cardinality $\omega_{1}$.

By a Borel space we understand a pair $(X, \mathscr{B})$, where $X$ is a set and $\mathscr{B}$ is a $\sigma$-algebra of its subsets. Inspired by topology and the theory of Boolean algebras, K. P. S. Bhaskara Rao and B. V. Rao in [2] started to build a theory of Borel spaces. Several results have been obtained, some questions were raised. One of them was the problem of constructing a rigid Borel space [2, P3, p. 21]. For a Borel space $(X, \mathscr{B})$ a bijection $f: X \rightarrow X$ is called automorphism (of $(X, \mathscr{B}))$ if for any $A \in \mathscr{B}, f(A)$ and $f^{-1}(A)$ belong to $\mathscr{B}$. A Borel space $(X, \mathscr{B})$ is rigid provided the identity function is the only automorphism of $(X, \mathscr{B})$. The first construction of such a space has been given by $\mathbf{R}$. M. Shortt and J. van Mill [4] but on the set of large cardinality. Another one presented by $\mathbf{M}$. Droste [3] works on any set of cardinality greater than $\omega_{1}$-the first uncountable cardinal. The question was raised about cardinality $\omega_{1}$. The aim of this note is to give an example of such a rigid Borel space. In fact this example was found in September 1988 and presented on Winter School in January 1989.

For our purposes the following definitions and facts are needed (see [1]). If $X$ is a set cardinality of which is uncountable and regular-say $\kappa$-then a collection $\mathscr{I}$ of subsets of $X$ is called ideal if (i) $X \notin \mathscr{I}$; (ii) $\{x\} \in \mathscr{I}$ for each $x \in X$; (iii) $C \subseteq A \in \mathscr{I}$ implies $C \in \mathscr{I}$; and (iv) $\mathscr{I}$ is closed with respect to unions of fewer than $\kappa$ of its elements. Our ideal is an abbreviation for commonly used "nonprincipal proper $\kappa$-complete ideal," but we follow the convention of [1] because we use one of its results. One can restrict attention to the case $\kappa=\omega_{1}$ and in which our ideal is just a nonprincipal proper $\sigma$-ideal. Suppose $\mathscr{I}$ is an ideal on $X$ and $f: X \rightarrow X$ is a function. $f$ is called $\mathscr{I}$ small if $f^{-1}(x) \in \mathscr{I}$ for each $x \in X$, in this case $f_{*}(\mathscr{F})=\left\{A \subseteq X: f^{-1}(A) \in\right.$ $\mathscr{F}\}$ is an ideal on $X$. Now we can define the Rudin-Keisler preordering on ideals:

$$
\begin{aligned}
& \mathscr{I} \leq_{R K} \mathscr{I} \text { iff } \mathscr{I}=f_{*}(\mathscr{I}) \text { for some } \mathscr{I} \text {-small function } \\
& f: X \rightarrow X \text { and } \mathscr{I}<_{R K} \mathscr{I} \text { iff } \mathscr{I} \leq_{R K} \mathscr{I} \text { and } \mathscr{I} \Varangle_{R K} \mathscr{I} .
\end{aligned}
$$

Received by the editors June 1, 1990.

1980 Mathematics Subject Classification (1985 Revision). Primary 28A05. 
The following theorem comes from [1]:

Theorem [1, Theorem 5.10]. For any regular cardinal $\kappa$, there is a sequence $\left(\mathscr{I}_{\alpha}: \alpha<\kappa\right)$ of ideals on $\kappa$ such that $\mathscr{I}_{\beta}<_{R K} \mathscr{I}_{\alpha}$ whenever $\alpha<\beta<\kappa$.

Now we are ready to construct a rigid Borel space. Put $X=\left\{\omega_{1}^{n}: n=\right.$ $0,1, \ldots\}$, the set of finite sequences with values in the first uncountable cardinal $\left(\omega_{1}^{0}\right.$ is the empty sequence). There is a natural partial order on $X$ such that $X$ forms a tree; namely, for $x \in \omega_{1}^{n}$ and $y \in \omega_{1}^{m}: x \leq y$ iff $n \leq m$ and $y \mid n=x$. The cardinality of $X$ is $\omega_{1}$ so by the theorem, we can pick for any $x \in X$ an ideal $\mathscr{I}(x)$ on $\omega_{1}$ such that if $x \neq y$, then $\mathscr{I}(x)<_{R K} \mathscr{I}(y)$ or $\mathscr{I}(y)<_{R K} \mathscr{I}(x)$. For $x \in X$ let $\operatorname{Ims}(x)=\{y \in X: y$ is an immediate successor of $x\} \cup\{x\}$. Any $\operatorname{Ims}(x)$ has cardinality $\omega_{1}$ so we may consider $\mathscr{I}(x)$ as an ideal on $\operatorname{Ims}(x)$. Further for $x \in X$ and $A \in \mathscr{I}(x)$, let $T(x)=\{y \in X: x \leq y\}$ and $T(x, A)=T(x) \backslash\{T(y): y \in A\}$. Let $\mathscr{B}$ be the least $\sigma$-algebra of subsets of $X$ containing the family $\{T(x, A): x \in X$, $A \in \mathscr{F}(x)\}$. It is easily seen that for any two distinct $x, y \in X$, at least one of $T(x)$ or $T(y)$ contains exactly one of these elements. We have also

if $x \in B \in \mathscr{B}$, then there is an $A \in \mathscr{I}(x)$ such that $x \notin A$ (*) $\quad$ and $T(x \cdot A) \subseteq B$ (for any fixed $x$ the family of sets satisfying (*) forms a $\sigma$-algebra and contains all of $T(y, C)$ 's).

Suppose for contradiction that $(X, \mathscr{B})$ is not rigid, i.e. there exists $f: X \rightarrow$ $X$ an automorphism but not the identity. Fix $p, q \in X, p \neq q$ such that $f(p)=q$. Due to $(*)$ we can inductively pick two sequences of sets $P_{k} \in \mathscr{I}(p)$ and $Q_{k} \in \mathscr{I}(q)$ not containing $p$ and $q$ respectively such that $P_{0}=\varnothing$, $f\left(T\left(p, P_{k}\right)\right) \supseteq T\left(q, Q_{k}\right)$ and $f^{-1}\left(T\left(q, Q_{k}\right)\right) \supseteq T\left(p, P_{k+1}\right)$. Thus for $P=$ $\bigcup\left\{P_{k}: k=0,1, \ldots\right\}$ and $Q=\bigcup\left\{Q_{k}: k=0,1, \ldots\right\}$ we have

$$
f(T(p, P))=T(q, Q) \neq \varnothing \quad \text { where } P \in \mathscr{I}(p) \text { and } Q \in \mathscr{I}(q) \text {. }
$$

Now we may define two functions $g: \operatorname{Ims}(p) \rightarrow \operatorname{Ims}(q)$ and $h: \operatorname{Ims}(q) \rightarrow$ $\operatorname{Ims}(p)$ in the following way. For any $x \notin P \cup\{p\}$, let $g(x)$ be the only element $y \in \operatorname{Ims}(q)$ satisfying $q<y \leq f(x)$ and for $x \in P \cup\{p\}$, let $g(x)=q$. Similarly we define the function $h$. The functions $g$ and $h$ are well defined due to $(* *)$.

Let us consider any $A \in \mathscr{I}(q)$. If we repeat the induction proceeding (**) but starting with $Q_{0}=A \cup Q \backslash\{q\}$ we obtain $P^{\prime} \in \mathscr{I}(p)$ and $Q^{\prime} \in \mathscr{I}(q)$, $p \notin P^{\prime}, q \notin Q^{\prime}$ and $f\left(T\left(p, P^{\prime}\right)\right)=T\left(q, Q^{\prime}\right)$. Thus $h(A)$ and $g^{-1}(A)$ are subsets of $P^{\prime} \cup\{p\} \in \mathscr{I}(p)$. By arbitrariness of $A$, we have $\mathscr{I}(q) \subseteq g_{*}(\mathscr{I}(p))$ and $h_{*}(\mathscr{I}(q)) \subseteq \mathscr{I}(p)$. Similarly we may obtain $\mathscr{I}(p) \subseteq h_{*}(\mathscr{I}(q))$ and $g_{*}(\mathscr{I}(p)) \subseteq \mathscr{I}(q)$. Finally $h_{*}(\mathscr{I}(q))=\mathscr{I}(p)$ and $g_{*}(\mathscr{I}(p))=\mathscr{I}(q)$. But this contradicts our choice of ideals and then ends the proof.

Remark. Modifying the proof of Baumgartner-Taylor-Wagon's Theorem one can obtain, for any regular $\kappa$, a family of ideals of size $2^{\kappa}$ noncomparable in 
the $\leq_{R K}$-preordering. Then as above one can construct on $\kappa$ a family of $2^{2^{\kappa}}$ rigid pairwise nonisomorphic Borel spaces.

\section{REFERENCES}

1. J. E. Baumgartner, A. D. Taylor, and S. Wagon, Structural properties of ideals, Dissertationes Math. (Rozprawy Mat.) 197 (1982).

2. K. P. S. Bhaskara Rao and B. V. Rao, Borel spaces, Dissertationes Math. (Rozprawy Mat.) 190 (1981).

3. M. Droste, The existence of rigid measurable spaces, Topology Appl. 31 (1989), 187-195.

4. R. M. Shortt and J. van Mill, Automorphism group for measurable spaces, Topology Appl. 30 (1988), 27-42.

Institute of Mathematics, Polish Academy of Sciences, Ul. Kopernika 18, 51-617 Wroclaw, Poland 